\title{
Perfil lipídico e inhibidores de integrasa: revisión sistemática y
} metaanálisis

\section{Lipid profile and integrase inhibitors: a systematic review and metaanalysis}

Jorge Alejandro Lopera-Rodríguez ${ }^{1 凶}$, Lucas López-Quiceno $^{2}$

${ }^{1}$ Magíster en Epidemiología, Grupo de Investigación e Innovación Biomédica, Instituto Tecnológico Metropolitano, Medellín.

2 Magíster en Epidemiología, Clínica CES, Medellín.

Fecha correspondencia:

Recibido: diciembre 17 de 2020.

Revisado: mayo 13 de 2021.

Aceptado: junio 21 de 2021.

Forma de citar:

Lopera-Rodríguez JA, López-

Quiceno L. Perfil lipídico e

inhibidores de integrasa: revisión

sistemática y metaanálisis. Rev

CES Med. 2021; 35(2): 77-97.

10.21615/cesmedicina.6017

\section{Open access}

\section{Derecho de autor}

Licencia creative commons

Ética de publicaciones

Revisión por pares

Gestión por Open Journal System DOI: http://dx.doi.org/10.21615/ cesmedicina.6017

ISSNe 2215-9177

ISSN 0120-8705

Publica con nosotros

\section{Resumen}

Introducción: en pacientes con virus de inmunodeficiencia humana algunos antirretrovirales afectan el perfil lipídico incrementando el riesgo cardiovascular. Hay evidencia de que los inhibidores de integrasa afectan poco al perfil lipídico. El presente estudio buscó evaluar la mejor evidencia disponible sobre cambios en lípidos de pacientes con virus de inmunodeficiencia humana que cambiaron su terapia antirretroviral a esquemas con inhibidores de integrasa. Métodos: revisión sistemática de la literatura con intención metaanalítica. A partir de la pregunta: "En pacientes mayores de 16 años con virus de inmunodeficiencia humana, los esquemas antirretrovirales que incluyen inhibidores de integrasa comparados con aquellos esquemas antirretrovirales que no los incluyen, ¿presentan cambios en el perfil lipídico?" se extrajeron palabras clave para búsqueda de la evidencia publicada entre 1997 y diciembre 2019. Se incluyeron estudios experimentales y observacionales y su calidad fue evaluada. Se realizó análisis por inhibidor de integrasa y parámetro lipídico buscándose síntesis cuantitativa de la evidencia. Resultados: se identificaron 17 estudios relevantes susceptibles de síntesis de la evidencia con un total de 5683 pacientes. De estos, 2878 entraron a síntesis cuantitativa. Acorde a lo encontrado, los 
Mayo - agosto de 2021

inhibidores de integrasa presentan mejor perfil lipídico comparados a otros antirretrovirales. Dolutegravir fue el que mostró mejor perfil lipídico cuando la comparación se hizo con inhibidores de proteasa. Raltegravir tuvo mejor perfil lipídico comparándolo con inhibidores de transcriptasa inversa no análogos de nucleósidos. Conclusiones: el uso de inhibidores de integrasa es un factor relevante en el control del riesgo cardiovascular en pacientes con virus de inmunodeficiencia humana.

Palabras clave: VIH; Inhibidores de integrasa; Colesterol; Dislipidemias; Revisión Sistemática.

\section{Abstract}

Introduction: some antiretrovirals affect the lipid profile in human immunodeficiency virus patients increasing their cardiovascular risk. Integrase inhibitors generate little lipid alteration. The present study evaluated the best available evidence about changes in the lipid profile in human immunodeficiency virus patients who had switch from different antiretroviral therapies to schemes with integrase inhibitors. Methods: a systematic review with meta-analytic intention was carried out. From the question "How does antiretroviral schemes with integrase inhibitors impact in lipid profile in human immunodeficiency virus patients compared to antiretroviral schemes without integrase inhibitors?" an evidence search was done. Articles from experimental and observational studies were included and the quality was evaluated. An analysis by integrase inhibitor and lipid parameters was performed. Results: 17 relevant studies were identified and 2878 patients were included in the quantitative synthesis. According to evidence, integrase inhibitors had a better lipid profile compared to other antiretrovirals. Dolutegravir had a better metabolic profile when it was compared with protease inhibitors. Raltegravir had a better lipid profile when it was compared to non-nucleoside analog reverse transcriptase inhibitors. Conclusions: integrase inhibitors are a relevant factor for cardiovascular risk control in patients with human immunodeficiency virus.

Keywords: HIV; Integrase inhibitors; Cholesterol; Dyslipidemia; Systematic review.

\section{Introducción}

Gracias a la terapia antirretroviral y políticas internacionales se ha logrado un descenso en la mortalidad por VIH desde $2005^{(1)}$ que ha redundado en una población con VIH más envejecida. Por otro lado, el riesgo cardiovascular en esta población es mayor. Dicho riesgo fluctúa entre $40 \%$ a 75 más que la población general ${ }^{(2,3)}$ y se ha asociado a aterosclerosis, inflamación 
crónica, activación inmune y terapia antirretroviral(4). El estudio Data Collection on Adverse Events of Anti-HIV Drugs evidencia que los antirretrovirales son un factor individual para el incremento del riesgo cardiovascular ${ }^{(5)}$.

Los inhibidores de proteasa (IP) son los antirretrovirales que generan mayor alteración lipídica ${ }^{(6)}$. Por el contrario, los inhibidores de integrasa han mostrado un mejor comportamiento lipídico, particularmente dolutegravir ${ }^{(7)}$. En el intermedio están los inhibidores de transcriptasa inversa análogos de nucleósidos/nucleótidos (ITIAN) y no análogos (ITINN). Sin embargo, los inhibidores de entrada presentan el mejor comportamiento lipídico ${ }^{(8)}$.

Conocer el impacto en el perfil lipídico de cada antirretroviral puede ser clave al escoger el esquema de tratamiento, particularmente en pacientes con alto riesgo cardiovascular. Para esto, se hace necesaria la síntesis periódica de la evidencia. Entre las síntesis previas, se encuentran los metaanálisis de Patel et al. ${ }^{(9)}$ y de Snedecor et al. ${ }^{(7)}$, quiénes han confirmado el efecto benigno de los inhibidores de integrasa sobre el perfil lipídico, aunque estos han sido enfocados sobre pacientes naive. El presente estudio buscó evaluar la mejor evidencia disponible sobre el perfil lipídico en pacientes con VIH que hicieron cambio de su terapia antirretroviral hacia esquemas con inhibidores de integrasa, comparándolos con aquellos que no. Además de incluir evidencia relacionada con dolutegravir, raltegravir y elvitegravir, como los metaanálisis citados previamente, se incluyó también evidencia relacionada con bictegravir.

\section{Materiales y métodos}

Este estudio fue una revisión sistemática con intención metaanalítica siguiendo el manual Cochrane. El protocolo fue registrado en PROSPERO con código CRD42020147393. Se identificaron palabras clave a partir de la pregunta: "En pacientes mayores de 16 años con virus de inmunodeficiencia humana, los esquemas antirretrovirales que incluyen inhibidores de integrasa comparados con aquellos esquemas antirretrovirales que no los incluyen, ¿presentan cambios en el perfil lipídico?". Las palabras clave utilizadas en la búsqueda de la literatura fueron: HIV, human immunodeficiency virus, integrase inhibitors, raltegravir, elvitegravir, dolutegravir, bictegravir, cholesterol, dyslipidemia, triglycerides, high density cholesterol, low density cholesterol, clinical trials, cohort, case and controls. Estas se organizaron en sintaxis de búsqueda adaptadas para cada repositorio. La búsqueda incluyó publicaciones desde 1997, año de inclusión del término Integrase Inhibitors en Medline, hasta 20 de diciembre de 2019, fecha de la última búsqueda. Esta se realizó en PubMed, Scopus, OVID, LILACS y CENTRAL aplicando 
Mayo - agosto de 2021

terminología MeSH, DeCS y búsqueda libre de términos. También incluyó repositorios como Clinical Trials, y literatura gris en Google Scholar. Se incluyeron estudios en inglés, español, portugués, francés e italiano.

De los resultados, se seleccionaron artículos de estudios experimentales y observacionales que cumplieran los siguientes criterios de inclusión: población mayor de 16 años, con VIH y en terapia antirretroviral. Que evaluaran al menos uno de los siguientes parámetros: colesterol total, colesterol LDL, colesterol HDL o triglicéridos. Al menos dos mediciones: antes y durante el tratamiento, en mínimo 12 semanas. Fueron excluidos estudios sin valores de medición de lípidos. La selección se realizó por dos evaluadores de forma independiente. Las diferencias en esta fueron dirimidas por consenso. Los datos faltantes se solicitaron a los autores vía correo electrónico.

Posteriormente se caracterizaron los estudios. Dos investigadores de forma independiente evaluaron la calidad y riesgo de sesgo con las herramientas RoB $2.0^{(10)}$ para estudios experimentales y ROBINS-| ${ }^{(11)}$ para estudios observacionales. Las diferencias en la evaluación fueron dirimidas por un tercer evaluador.

Las medidas reportadas en medianas se convirtieron a medias basándose en la técnica propuesta por Wan et al. ${ }^{(12)}$. Los errores estándar fueron convertidos a desviaciones estándar dividiéndolos entre la raíz cuadrada de la muestra. Las unidades reportadas en $\mathrm{mmol} / \mathrm{L}$ se convirtieron a $\mathrm{mg} / \mathrm{dL}$ así: para colesterol total, LDL y HDL el valor reportado se dividió entre 0,02586 y para triglicéridos entre 0,01129 . En estudios con resultados en gráficas, los datos se extrajeron usando la herramienta WebPlotDigitizer 4.2 ${ }^{(13)}$.

Inicialmente, se hizo un análisis exploratorio con forest plot para identificar tendencias. Posteriormente, se realizó síntesis cuantitativa con aquellos estudios que fueran comparables entre sí. La evidencia se sintetizó por modelo de efectos aleatorios buscando manejar la heterogeneidad entre estudios que no fuera observable. Se estimó la medida de síntesis con intervalo de confianza del $95 \%$ e intervalo de predicción del $95 \%$. La heterogeneidad entre estudios fue cuantificada por Tau de forma relativa e $\mathrm{I}^{2}$ de forma absoluta. La síntesis cualitativa de la evidencia se realizó siguiendo la guía SMF-ICONS-Quant ${ }^{(14)}$. Para análisis de sensibilidad se usó la metodología de gráfico de embudo, teniendo como criterio de realización mínimo diez estudios por metaanálisis. 
La aprobación ética y consentimiento informado de pacientes no fueron requeridos dada la naturaleza del presente estudio.

\section{Resultados}

El diagrama de flujo de resultados de búsqueda y selección de la evidencia puede verse en la figura 1.

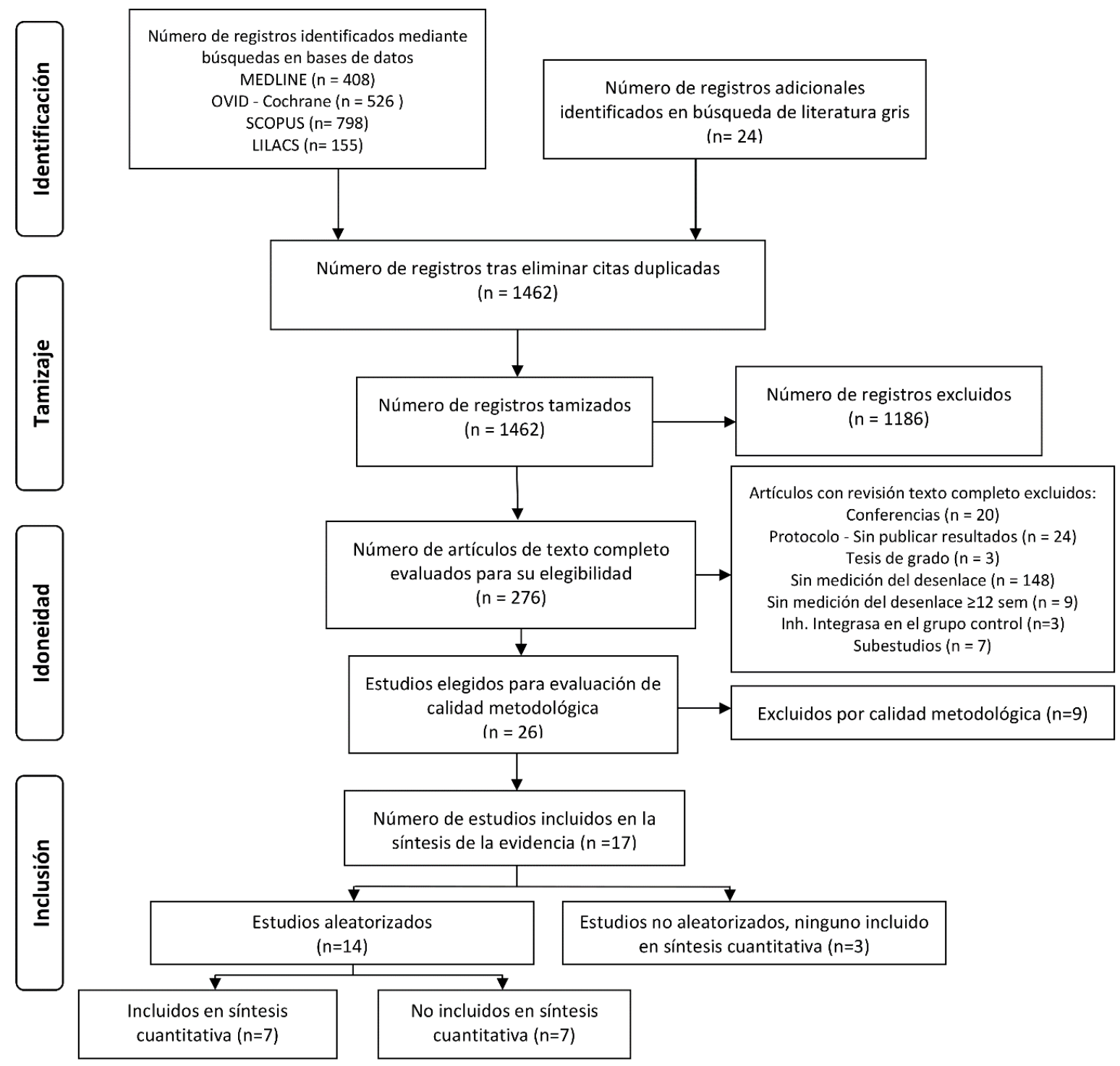

Figura 1. Diagrama de flujo de la selección de los estudios. 
Los 26 estudios seleccionados para evaluación de calidad se publicaron entre 2009 y 2019, de los cuales 20 fueron ensayos clínicos aleatorizados abiertos, un ensayo clínico fue cegado y otro cegado en las primeras 48 semanas y abierto en semanas posteriores. Se identificaron tres estudios de cohorte y uno de casos y controles. Entre los ensayos clínicos, 14 fueron de no inferioridad, uno de superioridad y el resto no lo describió. Dentro de estos, 15 tuvieron un enfoque de análisis por intención a tratar y tres por protocolo, el resto no lo describió. En estos últimos, se buscó deducir el análisis utilizando el texto (ver anexo).

En 14 estudios evaluaron esquemas con raltegravir, seis con dolutegravir, cinco con elvitegravir y uno con bictegravir. La comparación más frecuente fue con esquemas con inhibidores de proteasa potenciados con ritonavir (IP/r) en 11 estudios, con ITINN en siete, IP/r+ITIAN en cinco, inhibidores de entrada en uno y el resto no lo describió. En 15 estudios se evaluaron parámetros lipídicos a las 48 semanas, $44 \%$ reportó uso de hipolipemiantes como estatinas entre $0 \%$ a $30 \%$ y $56 \%$ de los estudios no reportó dicho uso. La población de estos estudios estuvo entre 30 y 60 años. En 18 estudios se incluyó $2 \%$ a 51 \% de población femenina y sólo dos incluyeron población 100 \% femenina, el resto no lo describió. El 80 \% de los estudios garantizó supresión viral al ingreso, mientras que 16 \% no lo garantizó y 4 \% no lo describió.

\section{Evaluación de calidad}

De los 15 estudios con análisis por intención a tratar, las principales fuentes de riesgo de sesgo fueron desviaciones en la intervención, datos de resultados perdidos y selección de resultados reportados (figura 2). Fueron excluidos cinco estudios por estas razones.

En los siete estudios con análisis por protocolo las principales fuentes de riesgo de sesgo fueron desviaciones en la intervención, datos de resultados perdidos y selección de resultados reportados (figura 2). Tres estudios fueron excluidos por estas razones. En los tres estudios de cohorte y en el de casos y controles, el principal riesgo de sesgo fue la selección en reporte de resultados (figura 2). Un estudio fue excluido por riesgo serio de sesgo por ausencia en la descripción del manejo de variables de confusión.

Los 17 estudios identificados con buena calidad sumaron una población de 5683 participantes y en estos se exploró tendencias. Siete estudios fueron viables para síntesis cuantitativa de la evidencia incluyendo 2878 participantes. 


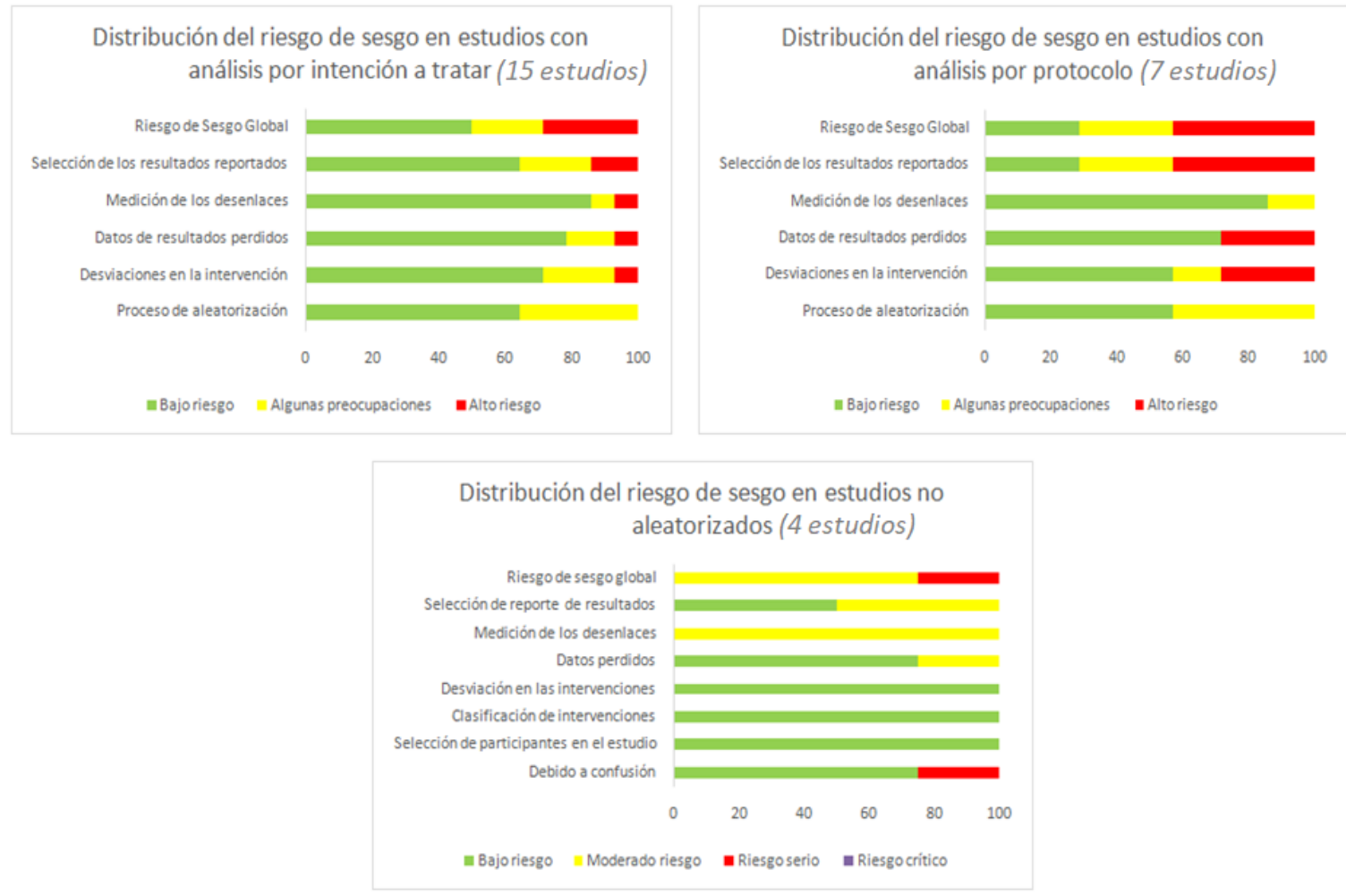

Figura 2. Evaluación del riesgo de sesgo.

\section{Raltegravir}

En los estudios de raltegravir ${ }^{(15-24)}$ se usó una dosis de $400 \mathrm{mg}$ cada 12 horas. Hubo seis esquemas de comparación: dos estudios, SWITCHMARK 1 y SWITCHMARK $2^{(17)}$, con comparaciones incluyendo IP/r, un estudio ${ }^{(24)}$ con comparación con ITINN, un estudio ${ }^{(16)}$ evaluó los comparadores ITINN e IP/r, un estudio ${ }^{(15)}$ evaluó la comparación enfuvirtide+ITINN, tres estudios $^{(18-20)}$ compararon raltegravir+IP/ $r$ con IP/ $r+I T I A N$ y un estudio comparó inhibidores de integrasa $+\mathrm{IP} / \mathrm{r}$ con terapia antirretroviral estándar ${ }^{(23)}$.

Según figura 3 , el colesterol total tiende a disminuir cuando se compara raltegravir con IP/r e ITINN, tiende a ser neutro o a incrementarse cuando se compara raltegravir+IP/r con IP/ $r+$ ITIAN, con la terapia antirretroviral estándar (sHAART) y con enfuvirtide+ITINN (ENF+ITINN). El colesterol LDL tiende a disminuir según Lake et al. ${ }^{(16)}$ y Gupta et al. ${ }^{(24)}$, tiene un comportamiento neutro cuando se compara con IP/ $\mathrm{r}$ y a incrementarse en el resto de comparaciones. En cuanto al colesterol HDL, en todos los estudios tiende hacia comportamiento neutro. Para triglicéridos, hay tendencia hacia disminución en la comparación con IP/r e ITINN, tendencia al incremento en el resto de escenarios. 


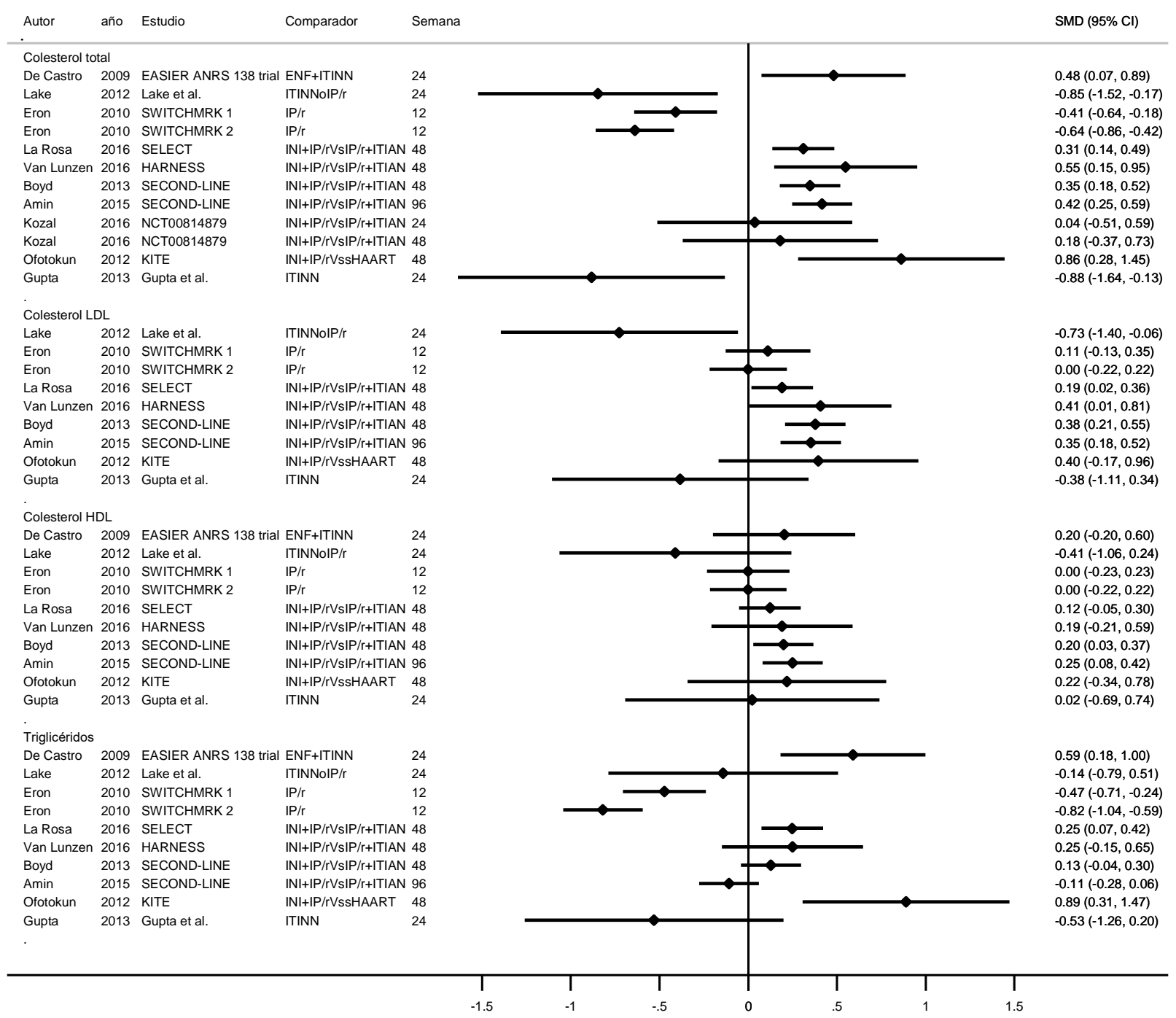

Figura 3. Forest plot de cambios en los lípidos de raltegravir vs otros antirretrovirales.

INI: inhibidor de integrasa

En el estudio de cohorte NEVRAST ${ }^{(25)}$ se comparó sustitución de terapia antirretroviral a esquemas con nevirapina o raltegravir comparándolos con rosuvastatina y evaluando el impacto sobre dislipidemia a 48 semanas. Cuando se realizó cambio hacia raltegravir o nevirapina hubo mejoría de parámetros lipídicos, especialmente triglicéridos. Sin embargo, con rosuvastatina la mejoría del colesterol total y LDL fue mayor que con raltegravir y nevirapina, pero no en colesterol HDL, donde raltegravir tuvo mejor desempeño. 
En el estudio de casos y controles de Olalla $2012^{(26)}$ en aquellos pacientes con terapia antirretroviral con raltegravir, tras seis meses, hubo un descenso del colesterol HDL y triglicéridos en los controles.

\section{Dolutegravir}

En estudios con dolutegravir ${ }^{(27-29)}$ la dosis usada fue de $50 \mathrm{mg} /$ día. Se encontró que el colesterol total, colesterol LDL y triglicéridos disminuyeron para dolutegravir comparado a IP/r (figura 4); sin embargo, en el metaanálisis para colesterol total y triglicéridos, se encontró una alta heterogeneidad y un intervalo de predicción amplio (figura 5). Para colesterol HDL, en el estudio de Aboud $2019^{(29)}$ se evidenció un leve incremento al inicio de la terapia manteniéndose en equilibrio. En el metaanálisis sobre este parámetro no se identificó una tendencia (figura 5).

El estudio de cohorte SCOLTA ${ }^{(30)}$ dividió a su población en dos grupos: cambio desde ITINN (efavirenz) +2 ITIAN y desde IP/r+2 ITIAN. A la vez, cada grupo se subdividió en tres subgrupos: terapia con dolutegravir, elvitegravir o rilpivirina. En relación a dolutegravir, hubo tendencia a disminución de colesterol total, colesterol LDL y triglicéridos, pero la rilpivirina presentó mejor perfil. 


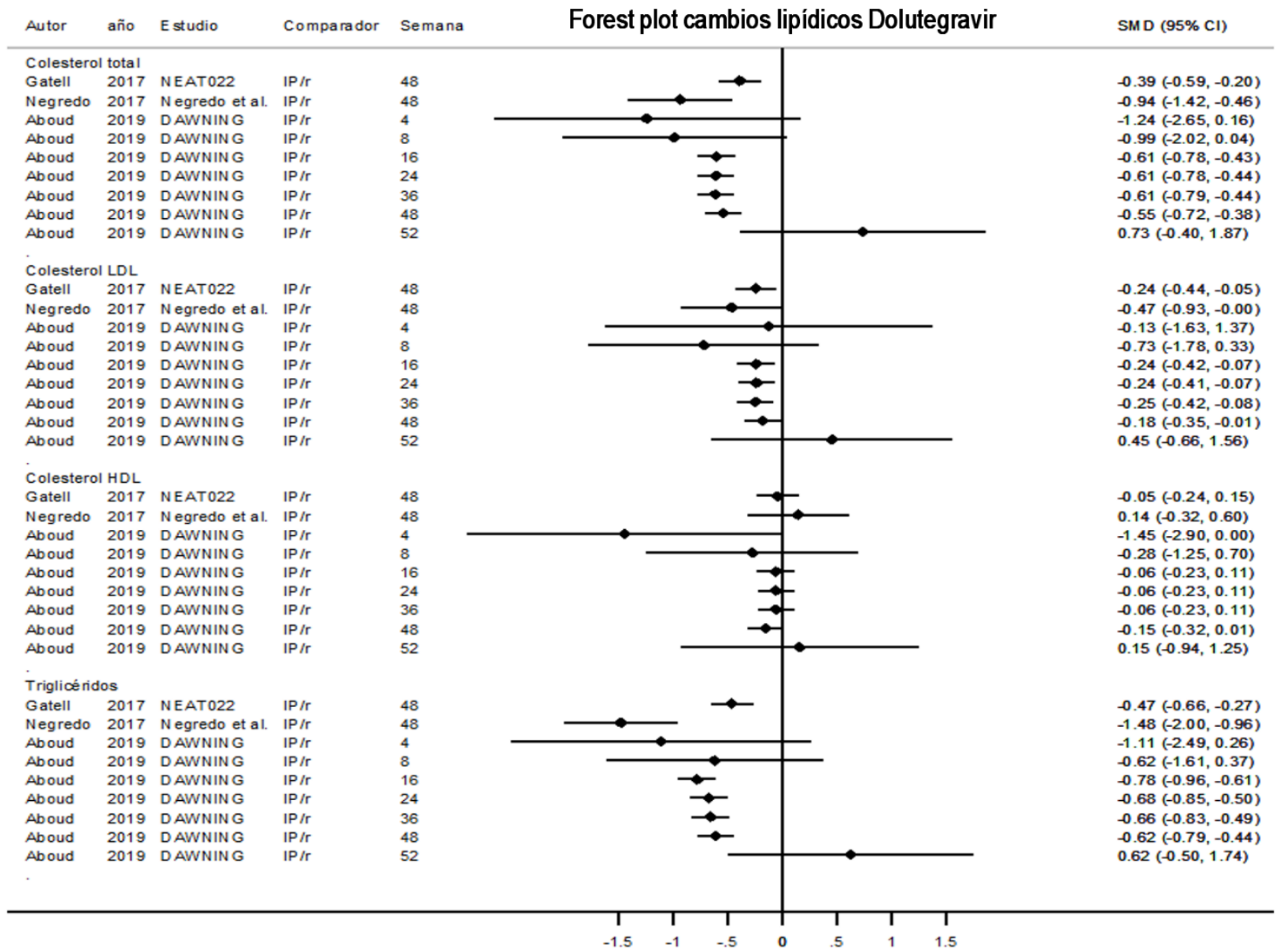

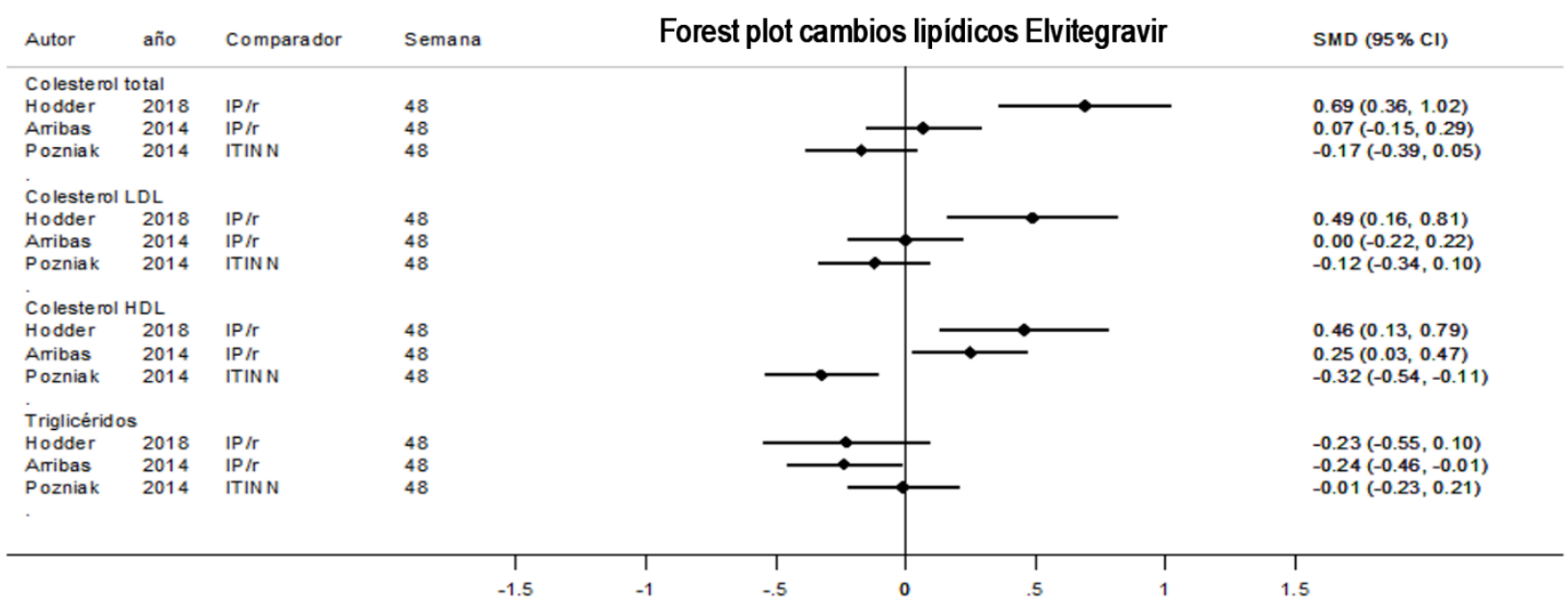

Figura 4. Forest plot de cambios en los lípidos de dolutegravir y elvitegravir vs otros antirretrovirales. INI: inhibidor de integrasa 


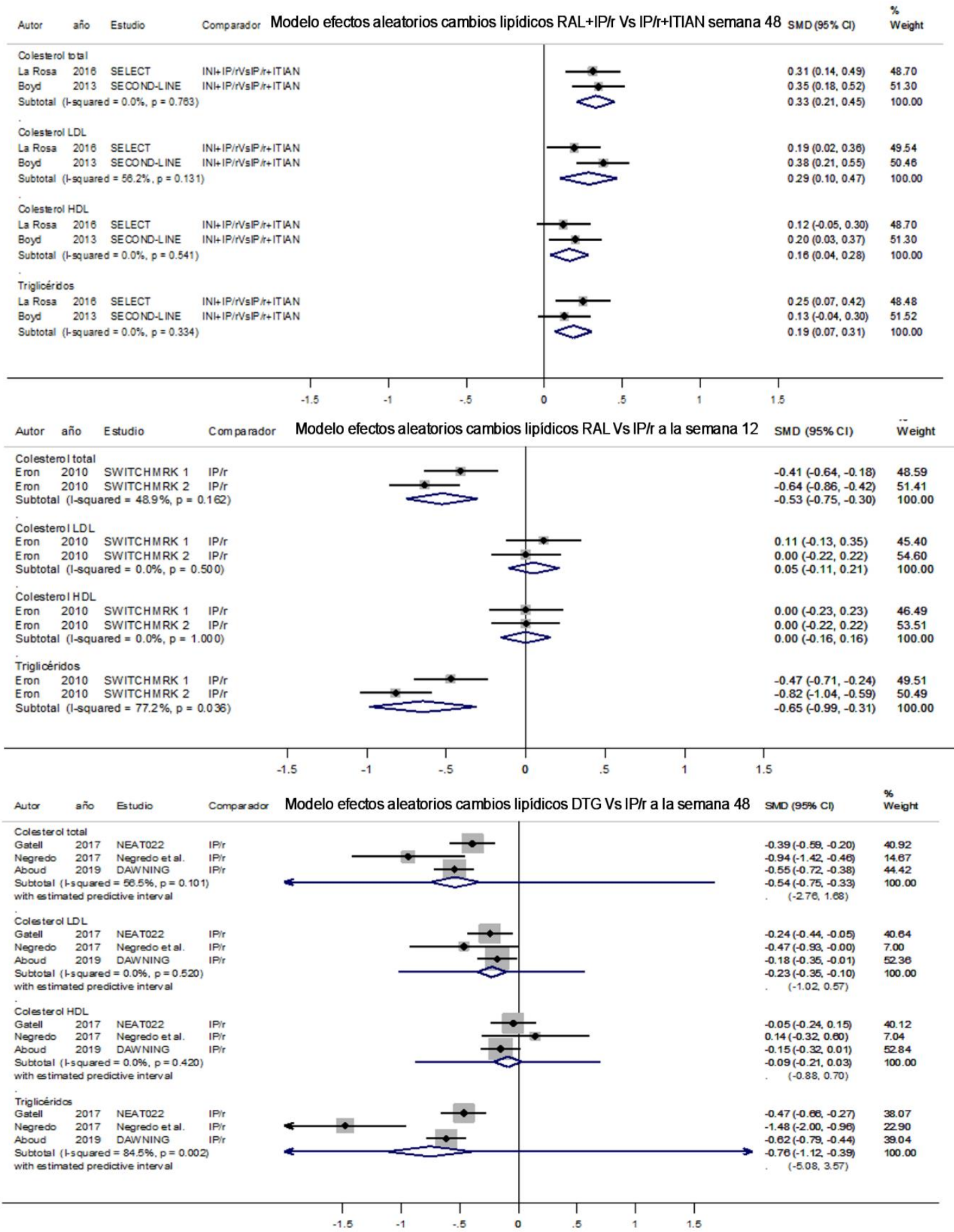

Figura 5. Metaanálisis posibles para raltegravir y dolutegravir.

Total pacientes metanálisis RAL+IP/r Vs IP/r+ITIAN: 1056. Total pacientes metanálisis RAL Vs IP/r: 707. Total pacientes metanálisis DTG Vs IP/r: 1115 . Total pacientes en síntesis cuantitativa:

2878. RAL: Raltegravir. DTG: Dolutegravir.

$\mathrm{NI}$ : inhibidor de integrasa 
Mayo - agosto de 2021

\section{Elvitegravir}

En los estudios incluidos ${ }^{(31-33)}$ el elvitegravir se combinó con cobicistat y se usó a dosis de $150 \mathrm{mg} /$ día. En la comparación con ITINN, hubo tendencia hacia disminución del colesterol total, LDL y HDL y un comportamiento neutral para triglicéridos a semana 48. Cuando se comparó elvitegravir con IP/r, hubo una tendencia al incremento del colesterol total, LDL y HDL y una tendencia hacia disminución de triglicéridos (figura 4). En el estudio de Taramasso en $2018^{(30)}$, el colesterol total y LDL tendieron hacia la neutralidad, mientras que los triglicéridos tendieron al incremento. Rilpivirina presentó un mejor perfil lipídico que elvitegravir.

\section{Bictegravir}

Sólo se encontró un estudio para bictegravir ${ }^{(34)}$. En este, se usó dosis de $50 \mathrm{mg} /$ día y el colesterol total fue más bajo en el grupo de intervención que en el de comparación a la semana 48. Esto mismo pasó con colesterol LDL. En cuanto a colesterol HDL, hubo una leve tendencia hacia el incremento. Por otro lado, se identificó una disminución de triglicéridos para el grupo con bictegravir.

\section{Discusión}

En el escenario de comparación inhibidores de integrasa con $I P / r$, dolutegravir es aquel inhibidor de integrasa con mejor perfil lipídico, seguido por raltegravir, bictegravir y elvitegravir. Sin embargo, en el escenario de comparación inhibidores de integrasa con ITINN, raltegravir presenta mejor perfil lipídico, seguido de elvitegravir y dolutegravir, sin evidencia para bictegravir. Por otro lado, la combinación de inhibidores de integrasa $+I P / r$ posee un pobre perfil lipídico cuando se compara con IP/r+ITIAN, haciendo poco recomendable dicha combinación.

Los hallazgos del presente estudio van en consonancia con metaanálisis previos ${ }^{(7,9,35)}$. En el metaanálisis en red de Patel et al. de $2014^{(9)}$, dolutegravir mostró una disminución significativa en incrementos de colesterol total, LDL y HDL, comparado con efavirenz, elvitegravir/cobicistat y atazanavir, lopinavir y darunavir potenciados con ritonavir. Sin embargo, dichos cambios no difirieron a lo encontrado con raltegravir y rilpivirina.

Quercia et al. en $2015^{(35)}$ realizan un análisis comparativo a partir de los estudios SPRING-1 ${ }^{(36)}$, SPRING-2(37,38), SINGLE(39) y FLAMINGO ${ }^{(40)}$. En este se concluye que un efecto ampliamente neutral sobre lípidos apoya aún más la recomendación de incluir dolutegravir en la terapia antirretroviral inicial. 
Snedecor et al. en $2019^{(7)}$, realizan búsqueda sistemática y metaanálisis en red comparando dolutegravir con otros antirretrovirales en pacientes naive. Incluyó 36 estudios. Dolutegravir mostró una supresión viral más alta que los inhibidores de proteasa y que los ITINN y similar a Ios ITIAN. Dolutegravir tuvo mejor perfil lipídico en colesterol total, LDL y triglicéridos comparándose con efavirenz, elvitegravir/cobicistat y atazanavir, lopinavir y darunavir potenciados con ritonavir, pero no superior a rilpivirina, raltegravir y bictegravir. En cuanto a colesterol HDL, dolutegravir no mostró mejor comportamiento.

Lo anterior, puede hacer recomendable que en el contexto de un paciente con trastornos en su perfil lipídico y con esquema con IP/ $r$, se considere cambio hacia un esquema que incluya inhibidores de integrasa como parte del abordaje de su riesgo cardiovascular, el cual debería combinarse con dos ITIAN como tenofovir/emtricitabina. Sin embargo, dicha conducta no debería reemplazar otras como el uso de estatinas.

\section{Limitaciones}

El presente estudio incluyó artículos en idiomas inglés, español, francés, portugués e italiano, excluyendo los de otros idiomas. Hay poca evidencia sobre bictegravir. Muchos de los estudios encontrados no tenían reporte de resultados en sus artículos ni en otras fuentes. Adicionalmente, muchos estudios no reportaban sus resultados en la misma métrica. Finalmente, no fue posible evaluar sesgo de publicación o análisis de sensibilidad por metarregresión dada la poca cantidad de estudios incluidos en metaanálisis.

En conclusión y en base a la evidencia encontrada, dolutegravir es el inhibidor de integrasa con mejor perfil metabólico cuando la comparación se hace con IP/r, seguido de raltegravir, bictegravir y elvitegravir. El raltegravir es el inhibidor de integrasa que mejor perfil metabólico tiene cuando la comparación se hace con ITINN, seguido de elvitegravir y dolutegravir.

\section{Conflicto de intereses}

Los autores declaran que no tuvieron conflictos de intereses para la realización del presente estudio. Los resultados del presente estudio hacen parte de una tesis para optar al grado académico de magíster en Epidemiología.

\section{Bibliografía}

1. Joint United Nations Programme on HIV/AIDS. Global AIDS update 2016. Geneva: UNAIDS. 2016 
2. Silverberg MJ, Leyden WA, Xu L, Horberg MA, Chao CR, Towner WJ, et al. Immunodeficiency and risk of myocardial infarction among HIV-positive individuals with access to care. JAIDS Journal of Acquired Immune Deficiency Syndromes. 2014;65(2):160-6.

3. Freiberg MS, Chang C-CH, Kuller LH, Skanderson M, Lowy E, Kraemer KL, et al. HIV infection and the risk of acute myocardial infarction. JAMA Internal Medicine. 2013;173(8):614-22.

4. Hsue PY, Waters DD. HIV infection and coronary heart disease: mechanisms and management. Nat Rev Cardiol. 2019;16(12):745-59.

5. Ryom L, Lundgren JD, El-Sadr W, Reiss P, Kirk O, Law M, et al. Cardiovascular disease and use of contemporary protease inhibitors: the $D$ : $A$ : $D$ international prospective multicohort study. The lancet HIV. 2018;5(6):e291-300.

6. Lundgren J, Mocroft A, Ryom L. Contemporary protease inhibitors and cardiovascular risk. Curr Opin Infect Dis. 2018;31(1):8-13.

7. Snedecor SJ, Radford M, Kratochvil D, Grove R, Punekar YS. Comparative efficacy and safety of dolutegravir relative to common core agents in treatment-naïve patients infected with HIV-1: a systematic review and network meta-analysis. BMC Infect Dis. 2019;19(1):484.

8. European AIDS Clinical Society. EACS Guidelines 10.0. 2019.

9. Patel DA, Snedecor SJ, Tang WY, Sudharshan L, Lim JW, Cuffe R, et al. 48-Week Efficacy and Safety of Dolutegravir Relative to Commonly Used Third Agents in Treatment-Naive HIV-1-Infected Patients: A Systematic Review and Network Meta-Analysis. PLoS One [Internet]. 2014 [citado 13 de mayo de 2020];9(9). Disponible en: https://www.ncbi.nlm.nih.gov/pmc/articles/PMC4154896/

10. Sterne JA, Savović J, Page MJ, Elbers RG, Blencowe NS, Boutron I, et al. RoB 2: a revised tool for assessing risk of bias in randomised trials. BMJ. 2019;366:14898.

11. Sterne JA, Hernán MA, Reeves BC, Savović J, Berkman ND, Viswanathan M, et al. ROBINSI: a tool for assessing risk of bias in non-randomised studies of interventions. BMJ. 2016;355:i4919. 
12. Wan X, Wang W, Liu J, Tong T. Estimating the sample mean and standard deviation from the sample size, median, range and/or interquartile range. BMC Medical Research Methodology. 2014;14(1):135.

13. Ankit Rohatgi. WebPlotDigitizer [Internet]. San Francisco, California, USA; 2019. Disponible en: https://automeris.io/WebPlotDigitizer

14. Campbell M, Katikireddi SV, Sowden A, McKenzie JE, Thomson H. Improving Conduct and Reporting of Narrative Synthesis of Quantitative Data (ICONS-Quant): protocol for a mixed methods study to develop a reporting guideline. BMJ open. 2018;8(2):e020064.

15. De Castro N, Braun J, Charreau I, Pialoux G, Cotte L, Katlama C, et al. Switch from enfuvirtide to raltegravir in virologically suppressed multidrug-resistant HIV-1-infected patients: a randomized open-label trial. Clin Infect Dis. 2009;49(8):1259-67.

16. Lake JE, McComsey GA, Hulgan TM, Wanke CA, Mangili A, Walmsley SL, et al. A randomized trial of raltegravir replacement for protease inhibitor or non-nucleoside reverse transcriptase inhibitor in HIV-infected women with lipohypertrophy. AIDS Patient Care STDs. 2012;26(9):532-40.

17. Eron JJ, Young B, Cooper DA, Youle M, Dejesus E, Andrade-Villanueva J, et al. Switch to a raltegravir-based regimen versus continuation of a lopinavir-ritonavir-based regimen in stable HIV-infected patients with suppressed viraemia (SWITCHMRK 1 and 2): two multicentre, double-blind, randomised controlled trials. Lancet. de 2010;375(9712):396407.

18. La Rosa AM, Harrison LJ, Taiwo B, Wallis CL, Zheng L, Kim P, et al. Raltegravir in secondline antiretroviral therapy in resource-limited settings (SELECT): a randomised, phase 3, non-inferiority study. Lancet HIV. 2016;3(6):e247-258.

19. van Lunzen J, Pozniak A, Gatell JM, Antinori A, Klauck I, Serrano O, et al. Brief Report: Switch to Ritonavir-Boosted Atazanavir Plus Raltegravir in Virologically Suppressed Patients With HIV-1 Infection: A Randomized Pilot Study. J Acquir Immune Defic Syndr. 2016;71(5):538-43.

20. Boyd MA, Kumarasamy N, Moore CL, Nwizu C, Losso MH, Mohapi L, et al. Ritonavirboosted lopinavir plus nucleoside or nucleotide reverse transcriptase inhibitors versus ritonavir-boosted lopinavir plus raltegravir for treatment of. Lancet. 2013;381(9883):2091-9. 
21. Amin J, Boyd MA, Kumarasamy N, Moore CL, Losso MH, Nwizu CA, et al. Raltegravir noninferior to nucleoside based regimens in second-line therapy with lopinavir/ritonavir over 96 weeks: a randomised open label study for the treatment of HIV-1 infection. PLoS One. 2015;10(2):e0118228.

22. Kozal MJ. A Pilot Randomized, Open-Label Study Comparing the Safety and Efficacy of a Raltegravir Based NRTI Sparing Regimen [Internet]. clinicaltrials.gov; 2016 ene [citado 18 de febrero de 2021]. Report No.: NCT00814879. Disponible en: https://clinicaltrials.gov/ct2/show/NCT00814879

23. Ofotokun I, Sheth AN, Sanford SE, Easley KA, Shenvi N, White K, et al. A switch in therapy to a reverse transcriptase inhibitor sparing combination of lopinavir/ritonavir and raltegravir in virologically suppressed HIV-infected patients: a pilot randomized trial to assess efficacy and safety profile: the KITE study. AIDS Res Hum Retroviruses. 2012;28(10):1196-206.

24. Gupta SK, Mi D, Moe SM, Dube MP, Liu Z. Effects of switching from efavirenz to raltegravir on endothelial function, bone mineral metabolism, inflammation, and renal function: a randomized, controlled trial. J Acquir Immune Defic Syndr. 2013;64(3):27983.

25. Calza L, Magistrelli E, Colangeli V, Borderi M, Bussini L, Bon I, et al. Substitution of nevirapine or raltegravir for protease inhibitor vs. rosuvastatin treatment for the management of dyslipidaemia in HIV-infected patients on stable antiretroviral therapy (Nevrast study). Infect Dis. 2017;49(10):737-47.

26. Olalla J, Del Arco A, de la Torre J, Salas D, Prada JL, García-Alegría J. Raltegravir en pacientes con infección por el virus de la inmunodeficiencia humana y alto riesgo vascular. Med Clin (Barc). 2012;138(3):107-9.

27. Gatell JM, Assoumou L, Moyle G, Waters L, Johnson M, Domingo P, et al. Switching from a ritonavir-boosted protease inhibitor to a dolutegravir-based regimen for maintenance of HIV viral suppression in patients with high cardiovascular risk: AIDS. 2017;31(18):2503-14.

28. Negredo E, Estrada V, Domingo P, Gutierrez MDM, Mateo GM, Puig J, et al. Switching from a ritonavir-boosted $\mathrm{PI}$ to dolutegravir as an alternative strategy in virologically suppressed HIV-infected individuals. J Antimicrob Chemother. 2017;72(3):844-9. 
29. Aboud M, Kaplan R, Lombaard J, Zhang F, Hidalgo JA, Mamedova E, et al. Dolutegravir versus ritonavir-boosted lopinavir both with dual nucleoside reverse transcriptase inhibitor therapy in adults with HIV-1 infection in whom first-line therapy has failed (DAWNING): an open-label, non-inferiority, phase $3 \mathrm{~b}$ trial. Lancet Infect Dis. $2019 ; 19(3): 253-64$.

30. Taramasso L, Tatarelli P, Ricci E, Madeddu G, Menzaghi B, Squillace N, et al. Improvement of lipid profile after switching from efavirenz or ritonavir-boosted protease inhibitors to rilpivirine or once-daily integrase inhibitors: Results from a large observational cohort study (SCOLTA). BMC Infect Dis [Internet]. 2018;18(1). Disponible en: https://www.scopus.com/inward/record.uri?eid=2-s2.0-

85050717684\&doi=10.1186\%2fs12879-018-3268-

5\&partnerID=40\&md5=e02cb552150d086608625b3c61119e0b

31. Arribas JR, Pialoux G, Gathe J, Di Perri G, Reynes J, Tebas P, et al. Simplification to coformulated elvitegravir, cobicistat, emtricitabine, and tenofovir versus continuation of ritonavir-boosted protease inhibitor with emtricitabine and tenofovir in adults with virologically suppressed HIV (STRATEGY-PI): 48 week results of a randomised, openlabel, phase 3b, non-inferiority trial. The Lancet infectious diseases. 2014;14(7):581-9.

32. Hodder S, Squires K, Kityo C, Hagins D, Avihingsanon A, Kido A, et al. Efficacy and safety of switching to coformulated elvitegravir, cobicistat, emtricitabine, and tenofovir alafenamide (E/C/F/Taf) in virologically suppressed women. Journal of acquired immune deficiency syndromes. 2018;78(2):209.

33. Pozniak A, Markowitz M, Mills A, Stellbrink H-J, Antela A, Domingo $P$, et al. Switching to coformulated elvitegravir, cobicistat, emtricitabine, and tenofovir versus continuation of non-nucleoside reverse transcriptase inhibitor with emtricitabine and tenofovir in virologically suppressed adults with HIV (STRATEGY-NNRTI): 48 week results of a randomised, open-label, phase $3 b$ non-inferiority trial. The Lancet Infectious Diseases. 2014;14(7):590-9.

34. Daar ES, DeJesus E, Ruane P, Crofoot G, Oguchi G, Creticos C, et al. Efficacy and safety of switching to fixed-dose bictegravir, emtricitabine, and tenofovir alafenamide from boosted protease inhibitor-based regimens in virologically suppressed adults with HIV1: 48 week results of a randomised, open-label, multicentre, phase 3, non-inferiority trial. Lancet HIV. 2018;5(7):e347-56. 
35. Quercia R, Roberts J, Martin-Carpenter L, Zala C. Comparative Changes of Lipid Levels in Treatment-Naive, HIV-1-Infected Adults Treated with Dolutegravir vs. Efavirenz, Raltegravir, and Ritonavir-Boosted Darunavir-Based Regimens Over 48 Weeks. Clin Drug Investig. 2015;35(3):211-9.

36. Van Lunzen J, Maggiolo F, Arribas JR, Rakhmanova A, Yeni P, Young B, et al. Once daily dolutegravir (S/GSK1349572) in combination therapy in antiretroviral-naive adults with HIV: Planned interim 48 week results from SPRING-1, a dose-ranging, randomised, phase 2b trial. Lancet Infect Dis. 2012;12(2):111-8.

37. Raffi F, Jaeger H, Quiros-Roldan E, Albrecht H, Belonosova E, Gatell JM, et al. Once-daily dolutegravir versus twice-daily raltegravir in antiretroviral-naive adults with HIV-1 infection (SPRING-2 study): 96 week results from a randomised, double-blind, noninferiority trial. The Lancet infectious diseases. 2013;13(11):927-35.

38. Raffi F, Rachlis A, Stellbrink H-J, Hardy WD, Torti C, Orkin C, et al. Once-daily dolutegravir versus raltegravir in antiretroviral-naive adults with HIV-1 infection: 48 week results from the randomised, double-blind, non-inferiority SPRING-2 study. The Lancet. 2013;381(9868):735-43.

39. Walmsley SL, Antela A, Clumeck N, Duiculescu D, Eberhard A, Gutierrez F, et al. Dolutegravir plus abacavir-lamivudine for the treatment of HIV-1 infection. N Engl J Med. 2013;369(19):1807-18.

40. Clotet B, Feinberg J, Van Lunzen J, Khuong-Josses M-A, Antinori A, Dumitru I, et al. Oncedaily dolutegravir versus darunavir plus ritonavir in antiretroviral-naive adults with HIV1 infection (FLAMINGO): 48 week results from the randomised open-label phase $3 \mathrm{~b}$ study. The Lancet. 2014;383(9936):2222-31. 
Anexo. Descripción de los estudios seleccionados

\begin{tabular}{|c|c|c|c|c|c|c|c|c|c|c|c|c|}
\hline Nombre & Autor & Año & Diseño & Metodología & $\begin{array}{l}\text { Tipo de } \\
\text { análisis }\end{array}$ & $\begin{array}{c}\text { Edad en } \\
\text { mediana } \\
(R I C)+\end{array}$ & $\begin{array}{l}\text { Participantes } \\
\text { femeninos } \\
\text { (\%) }\end{array}$ & Esquema previo & $\begin{array}{c}\text { Supresión } \\
\text { virológica } \\
\text { de base }\end{array}$ & $\begin{array}{c}\text { INI } \\
\text { evaluado }\end{array}$ & Posología & $\begin{array}{c}\text { Otros ARV en } \\
\text { grupo } \\
\text { intervención }\end{array}$ \\
\hline $\begin{array}{l}\text { GS-US-380- } \\
1878\end{array}$ & Daar, ES & 2018 & $\begin{array}{l}\text { ECA open } \\
\text { label }\end{array}$ & $\begin{array}{c}\text { No } \\
\text { inferioridad }\end{array}$ & Protocolo & $48(20-74)$ & 16 & $\begin{array}{c}\text { Atazanavir/ro } \\
\text { Darunavir/r }+ \\
\text { Emtricitabina/tenofovir } \\
\text { DF o } \\
\text { abacavir/lamivudina }\end{array}$ & $\mathrm{Si}$ & Bictegravir & 50 mg/día & $\begin{array}{c}\text { Emtricitabina/ } \\
\text { tenofovir AF }\end{array}$ \\
\hline Macias et al. & Macias, J & 2017 & $\begin{array}{c}\text { ECA open } \\
\text { label }\end{array}$ & No describe & $\begin{array}{c}\text { No } \\
\text { describe }\end{array}$ & $\begin{array}{l}52(45-55) / \\
48(47-52)\end{array}$ & 18 & $\begin{array}{c}\text { Efavirenz }+ \\
\text { emtricitabine/tenofovir } \\
\text { DF o } \\
\text { abacavir/lamivudine }\end{array}$ & Sí & Raltegravir & $\begin{array}{l}400 \mathrm{mg} \\
\mathrm{c} / 12 \mathrm{~h}\end{array}$ & $\begin{array}{c}\text { Emtricitabine/ } \\
\text { tenofovir DF o } \\
\text { abacavir/lami } \\
\text { vudine }\end{array}$ \\
\hline Kozal et al. & Kozal, MJ & 2016 & $\begin{array}{l}\text { ECA open } \\
\text { label }\end{array}$ & No describe & $\begin{array}{c}\text { No } \\
\text { describe }\end{array}$ & $48(9,6)^{*}$ & 19 & IP/r + ITIAN & $\mathrm{Si}$ & Raltegravir & $\begin{array}{l}400 \mathrm{mg} \\
\mathrm{c} / 12 \mathrm{~h}\end{array}$ & Atazanavir/r \\
\hline SELECT & $\begin{array}{l}\text { La Rosa, } \\
\text { AM }\end{array}$ & 2016 & $\begin{array}{l}\text { ECA open } \\
\text { label }\end{array}$ & $\begin{array}{c}\text { No } \\
\text { inferioridad }\end{array}$ & $\begin{array}{l}\text { Intención } \\
\text { a tratar }\end{array}$ & $\begin{array}{l}39(34-44) / \\
38(33-43)\end{array}$ & 51 & ITIAN + ITINN & No & Raltegravir & $\begin{array}{l}400 \mathrm{mg} \\
\mathrm{c} / 12 \mathrm{~h}\end{array}$ & Lopinavir/r \\
\hline HARNESS & $\begin{array}{c}\text { Van } \\
\text { Lunzen, J }\end{array}$ & 2016 & $\begin{array}{l}\text { ECA open } \\
\text { label }\end{array}$ & No describe & $\begin{array}{l}\text { Intención } \\
\text { a tratar }\end{array}$ & 44 (37-49) & 18 & 2ITIAN + IP/r O ITINN & $\mathrm{Si}$ & Raltegravir & $\begin{array}{l}400 \mathrm{mg} \\
\mathrm{c} / 12 \mathrm{~h}\end{array}$ & Atazanavir/r \\
\hline Gupta et al. & Gupta, SK & 2013 & $\begin{array}{l}\text { ECA open } \\
\text { label }\end{array}$ & No describe & $\begin{array}{l}\text { Intención } \\
\text { a tratar }\end{array}$ & $\begin{array}{c}38(12) / \\
39(10,6)^{*}\end{array}$ & 10 & $\begin{array}{c}\text { Efavirenz }+ \\
\text { emtricitabine/tenofovir } \\
\text { DF }\end{array}$ & $\mathrm{Si}$ & Raltegravir & $\begin{array}{l}\text { 400mg } \\
\mathrm{c} / 12 \mathrm{~h}\end{array}$ & $\begin{array}{c}\text { Tenofovir/ } \\
\text { emtricitabine }\end{array}$ \\
\hline SPARE & $\begin{array}{l}\text { Nishijima, } \\
\qquad \mathrm{T}\end{array}$ & 2013 & $\begin{array}{l}\text { ECA open } \\
\text { label }\end{array}$ & No describe & Protocolo & $\begin{array}{c}44(37-51) / \\
39(34-45)\end{array}$ & 2 & $\begin{array}{c}\text { Lopinavir } / r+ \\
\text { tenofovirDF/emtricitabi } \\
\text { na }\end{array}$ & $\mathrm{Si}$ & Raltegravir & 800 mg/día & Darunavir/r \\
\hline $\begin{array}{l}\text { SECOND- } \\
\text { LINE }\end{array}$ & Boyd, MA & 2013 & $\begin{array}{l}\text { ECA open } \\
\text { label }\end{array}$ & $\begin{array}{c}\text { No } \\
\text { inferioridad }\end{array}$ & $\begin{array}{l}\text { Intención } \\
\text { a tratar }\end{array}$ & $\begin{array}{c}38,5(33- \\
44,5) / 38,4 \\
(32-44,5) \\
\end{array}$ & 45 & ITINN + 2 ITIAN & No & Raltegravir & $\begin{array}{l}\text { 400mg } \\
\mathrm{c} / 12 \mathrm{~h}\end{array}$ & Lopinavir/r \\
\hline Lake et al. & Lake, JE & 2012 & $\begin{array}{l}\text { ECA open } \\
\text { label }\end{array}$ & No describe & Protocolo & $43(37-49)$ & 100 & $\begin{array}{c}102 \text { ITIAN + ITINN o } \\
I P / r\end{array}$ & $\mathrm{Si}$ & Raltegravir & $\begin{array}{l}400 \mathrm{mg} \\
\mathrm{c} / 12 \mathrm{~h}\end{array}$ & 2 ITIAN \\
\hline KITE & Ofotokun, I & 2012 & $\begin{array}{l}\text { ECA open } \\
\text { label }\end{array}$ & No describe & $\begin{array}{l}\text { Intención } \\
\text { a tratar }\end{array}$ & $\begin{array}{c}46(9) / \\
48(12)^{*}\end{array}$ & 37 & 2 ITIAN + IP/r o ITINN & $\mathrm{Si}$ & Raltegravir & $\begin{array}{l}400 \mathrm{mg} \\
\mathrm{c} / 12 \mathrm{~h}\end{array}$ & Lopinavir/r \\
\hline SPIRAL & Martinez, E & 2010 & $\begin{array}{l}\text { ECA open } \\
\text { label }\end{array}$ & $\begin{array}{c}\text { No } \\
\text { inferioridad }\end{array}$ & $\begin{array}{l}\text { Intención } \\
\text { a tratar }\end{array}$ & $\begin{array}{l}44(41-50) / \\
45(40-50)\end{array}$ & 23 & $\begin{array}{c}\text { IP/r + } 2 \text { o } 3 \text { ARV (1 о } 2 \\
\text { ITIAN +/- ITINN) }\end{array}$ & $\mathrm{Si}$ & Raltegravir & No describe & 203 ARV \\
\hline $\begin{array}{c}\text { SWITCHMRK } \\
1 \text { y } 2\end{array}$ & Eron, JJ & 2010 & ECA & $\begin{array}{c}\text { No } \\
\text { inferioridad }\end{array}$ & Protocolo & $\begin{array}{c}43(37-48) / \\
42(36-49)\end{array}$ & 22 & Lopinavir/r $+\geq 2$ ITIAN & $\mathrm{Si}$ & Raltegravir & $\begin{array}{l}400 \mathrm{mg} \\
\mathrm{c} / 12 \mathrm{~h}\end{array}$ & $\geq 2$ ITIAN \\
\hline
\end{tabular}




\begin{tabular}{|c|c|c|c|c|c|c|c|c|c|c|c|c|}
\hline Nombre & Autor & Año & Diseño & Metodología & $\begin{array}{l}\text { Tipo de } \\
\text { análisis }\end{array}$ & $\begin{array}{c}\text { Edad en } \\
\text { mediana } \\
(R I C)+\end{array}$ & $\begin{array}{l}\text { Participantes } \\
\text { femeninos } \\
\text { (\%) }\end{array}$ & Esquema previo & $\begin{array}{l}\text { Supresión } \\
\text { virológica } \\
\text { de base }\end{array}$ & $\begin{array}{c}\text { INI } \\
\text { evaluado }\end{array}$ & Posología & $\begin{array}{c}\text { Otros ARV en } \\
\text { grupo } \\
\text { intervención }\end{array}$ \\
\hline $\begin{array}{c}\text { EASIER } \\
\text { ANRS } 138 \\
\text { trial }\end{array}$ & $\begin{array}{l}\text { De Castro, } \\
\mathrm{N}\end{array}$ & 2009 & $\begin{array}{l}\text { ECA open } \\
\text { label }\end{array}$ & $\begin{array}{c}\text { No } \\
\text { inferioridad }\end{array}$ & $\begin{array}{l}\text { Intención } \\
\text { a tratar }\end{array}$ & $48(44-54)$ & 15 & $\begin{array}{c}\text { Enfuvirtida }+\geq 1 \text { ITINN, } \\
\text { IP o ITINN }\end{array}$ & $\mathrm{Si}^{* *}$ & Raltegravir & $\begin{array}{l}400 \mathrm{mg} \\
\mathrm{c} / 12 \mathrm{~h}\end{array}$ & $\begin{array}{l}\geq 1 \text { ITINN, IP o } \\
\text { ITINN }\end{array}$ \\
\hline DAWNING & Aboud, M & 2019 & $\begin{array}{l}\text { ECA open } \\
\text { label }\end{array}$ & $\begin{array}{c}\text { No } \\
\text { inferioridad }\end{array}$ & $\begin{array}{l}\text { Intención } \\
\text { a tratar }\end{array}$ & $\begin{array}{l}37,5(9,1) / \\
38,7(9,4)^{*}\end{array}$ & 35 & 2 ITIAN + ITINN & No & Dolutegravir & 50 mg/día & 2 ITIAN \\
\hline ASPIRE & Taiwo, BO & 2018 & $\begin{array}{l}\text { ECA open } \\
\text { label }\end{array}$ & $\begin{array}{c}\text { No } \\
\text { inferioridad }\end{array}$ & Protocolo & $47(38-54)$ & 12 & No describe & $\mathrm{Si}$ & Dolutegravir & 50 mg/día & Lamivudina \\
\hline NEAT022 & Gatell, JM & 2017 & $\begin{array}{l}\text { ECA open } \\
\text { label }\end{array}$ & $\begin{array}{c}\text { No } \\
\text { inferioridad }\end{array}$ & $\begin{array}{l}\text { Intención } \\
\text { a tratar }\end{array}$ & $54(51-58)$ & 12 & $2 I T I A N+I P / r$ & $\mathrm{Si}$ & Dolutegravir & 50 mg/día & 2ITIAN \\
\hline $\begin{array}{l}\text { Negredo et } \\
\quad \text { al. }\end{array}$ & Negredo, E & 2017 & $\begin{array}{l}\text { ECA open } \\
\text { label }\end{array}$ & No describe & $\begin{array}{c}\text { No } \\
\text { describe }\end{array}$ & $\begin{array}{c}46,8(39,3- \\
53,8) / 49,2 \\
(45,7-53,9)\end{array}$ & 15 & $\begin{array}{l}\text { Abacavir/lamivudina }+ \\
\qquad \mathrm{IP} / \mathrm{r}\end{array}$ & $\mathrm{Si}$ & Dolutegravir & 50 mg/día & $\begin{array}{l}\text { Abacavir/ } \\
\text { lamivudina }\end{array}$ \\
\hline $\begin{array}{l}\text { STRATEGY- } \\
\text { PI }\end{array}$ & Arribas & 2017 & $\begin{array}{l}\text { ECA open } \\
\text { label }\end{array}$ & $\begin{array}{c}\text { No } \\
\text { inferioridad }\end{array}$ & $\begin{array}{c}\text { No } \\
\text { describe }\end{array}$ & $\begin{array}{l}41(33-48) / \\
40(35-47)\end{array}$ & 14 & $\begin{array}{c}\mathrm{IP} / \mathrm{r}+ \\
\text { emtricitabina/tenofovir }\end{array}$ & $\mathrm{Si}$ & Elvitegravir & 150 mg/día & $\begin{array}{l}\text { Cobistat, } \\
\text { emtricitabina/ } \\
\text { tenofovir DF }\end{array}$ \\
\hline $\begin{array}{l}\text { STRATEGY- } \\
\text { PI }\end{array}$ & Arribas, JR & 2014 & $\begin{array}{l}\text { ECA open } \\
\text { label }\end{array}$ & $\begin{array}{c}\text { No } \\
\text { inferioridad }\end{array}$ & $\begin{array}{l}\text { Intención } \\
\text { a tratar }\end{array}$ & $\begin{array}{l}41(33-48) / \\
40(35-47)\end{array}$ & 15 & $\begin{array}{c}\mathrm{IP} / \mathrm{r}+ \\
\text { emtricitabina/tenofovir }\end{array}$ & $\mathrm{Si}$ & Elvitegravir & 150 mg/día & $\begin{array}{c}\text { Cobistat, } \\
\text { emtricitabina/ } \\
\text { tenofovir DF }\end{array}$ \\
\hline $\begin{array}{l}\text { STRATEGY- } \\
\text { NNRTI }\end{array}$ & Pozniak, A & 2014 & $\begin{array}{l}\text { ECA open } \\
\text { label }\end{array}$ & $\begin{array}{c}\text { No } \\
\text { inferioridad }\end{array}$ & $\begin{array}{l}\text { Intención } \\
\text { a tratar } \\
\text { modificad } \\
0\end{array}$ & $\begin{array}{l}43(34-49) / \\
39(32-48)\end{array}$ & 7 & $\begin{array}{c}\text { ITINN + } \\
\text { tenofovir/emtricitabina }\end{array}$ & $\mathrm{Si}$ & Elvitegravir & 150 mg/día & $\begin{array}{l}\text { Cobistat, } \\
\text { emtricitabina/ } \\
\text { tenofovir DF }\end{array}$ \\
\hline $\begin{array}{l}\text { GS-US-183- } \\
105\end{array}$ & Zolopa, AR & 2010 & $\begin{array}{l}\text { ECA open } \\
\text { label }\end{array}$ & $\begin{array}{c}\text { No } \\
\text { inferioridad }\end{array}$ & $\begin{array}{l}\text { Intención } \\
\text { a tratar }\end{array}$ & $44 * * *$ & 10 & 2 ITIAN +/- enfuvirtida & No & Elvitegravir & $\begin{array}{l}\text { Grupo 1: } 20 \\
\text { mg/día, } \\
\text { grupo 2: } 50 \\
\text { mg/día, } \\
\text { grupo 3: } \\
125 \text { mg/día }\end{array}$ & $\begin{array}{l}\text { Ritonavir + } 2 \\
\text { ITIAN }\end{array}$ \\
\hline WAVES 2 & Hodder, S & 2018 & $\begin{array}{c}\text { ECA } \\
\text { cegado 1a } \\
\text { fase y ECA } \\
\text { open label } \\
\text { 2a fase }\end{array}$ & $\begin{array}{c}\text { No } \\
\text { inferioridad }\end{array}$ & Protocolo & $36(30-44)$ & 100 & $\begin{array}{c}\text { Atazanavir } / \mathrm{r}+ \\
\text { emtricitabina/tenofovir } \\
\text { DF }\end{array}$ & $\mathrm{Si}$ & Elvitegravir & 150 mg/día & $\begin{array}{c}\text { Cobistat/ } \\
\text { emtricitabina } \\
\text { /tenofovir AF }\end{array}$ \\
\hline
\end{tabular}




\begin{tabular}{|c|c|c|c|c|c|c|c|c|c|c|c|c|}
\hline Nombre & Autor & Año & Diseño & Metodología & $\begin{array}{l}\text { Tipo de } \\
\text { análisis }\end{array}$ & $\begin{array}{c}\text { Edad en } \\
\text { mediana } \\
(R I C)+\end{array}$ & $\begin{array}{c}\text { Participantes } \\
\text { femeninos } \\
\text { (\%) }\end{array}$ & Esquema previo & $\begin{array}{c}\text { Supresión } \\
\text { virológica } \\
\text { de base }\end{array}$ & $\begin{array}{c}\text { INI } \\
\text { evaluado }\end{array}$ & Posología & $\begin{array}{c}\text { Otros ARV en } \\
\text { grupo } \\
\text { intervención }\end{array}$ \\
\hline $\begin{array}{l}\text { Borghetti et } \\
\text { al. }\end{array}$ & $\begin{array}{l}\text { Borghetti, } \\
\text { A }\end{array}$ & 2019 & Cohortes & No describe & $\begin{array}{c}\text { No } \\
\text { describe }\end{array}$ & $\begin{array}{c}48(40-54) / \\
48(43-53) / \\
51(43-57)\end{array}$ & 30 & 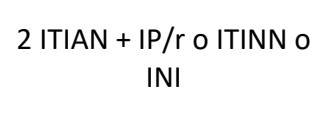 & $\mathrm{Si}$ & Dolutegravir & No describe & Lamivudina \\
\hline SCOLTA & $\begin{array}{c}\text { Taramasso, } \\
\mathrm{L}\end{array}$ & 2018 & Cohortes & No describe & $\begin{array}{c}\text { No } \\
\text { describe }\end{array}$ & $47,3(10,1)^{*}$ & 25 & $\begin{array}{c}2 \text { ITIAN + IP/r o } \\
\text { efavirenz }\end{array}$ & $\mathrm{Si}$ & $\begin{array}{c}\text { Dolutegravir } \\
\text { o } \\
\text { elvitegravir }\end{array}$ & No describe & 2ITIAN \\
\hline NEVRAST & Calza, L & 2017 & Cohortes & No describe & $\begin{array}{l}\text { Intención } \\
\text { a tratar }\end{array}$ & $46,6(8,8)^{*}$ & 21 & 2 ITIAN + IP/r & $\mathrm{Si}$ & Raltegravir & $\begin{array}{l}400 \mathrm{mg} \\
\mathrm{c} / 12 \mathrm{~h}\end{array}$ & 2 ITIAN \\
\hline Olalla et al. & Olalla J & 2012 & $\begin{array}{l}\text { Casos y } \\
\text { Controles }\end{array}$ & No describe & $\begin{array}{c}\text { No } \\
\text { describe }\end{array}$ & $\begin{array}{c}56,3(10,6) / \\
58,1(9,4)\end{array}$ & No describe & Variable & $\begin{array}{c}\text { No } \\
\text { describe }\end{array}$ & Raltegravir & No describe & Variable \\
\hline
\end{tabular}

+Datos reportados para cada grupo del estudio. En caso de reportarse un solo valor, corresponderá al valor global reportado por el estudio.

*Datos presentados en media (desviación estándar)

**Criterio de supresión virológica: niveles de RNA VIH $<400$ copias $/ \mathrm{mL}$ por $\geq 3$ meses, el resto de estudios cumplen con $<50$ copias $/ \mathrm{mL}$ por $\geq 3 \mathrm{meses}$.

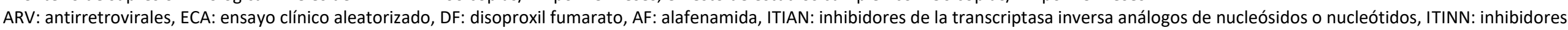
de la transcriptasa inversa no análogos de nucleósidos, IP: inhibidores de proteasa, r: potenciación con ritonavir, IE: inhibidores de entrada, INI: inhibidor de integrasa, NA: no aplica.

*** La medida de dispersión no fue reportada en el artículo del estudio. 